\title{
Saberes del oficio de enseñar en tensión: acerca de los velos que se corrieron en pandemia
}

\section{Knowledge of the profession of teaching in tension: about the veils that were released in a pandemic}

CARUSO, María Fabiana https://orcid.org/0000-0002-0853-6649

mfabicaruso@gmail.com

Facultad de Ciencias Sociales I UNICEN

\author{
ERROBIDART, Analía \\ https://orcid.org/0000-0002-6640-2462 \\ marina.yazyi@gmail.com \\ Facultad de Ciencias Sociales I UNICEN
}

\author{
GAITE, María Eugenia \\ https://orcid.org/0000-0002-3625-5350?lang=en \\ eugenia.gaite@gmail.com \\ Facultad de Ciencias Sociales | UNICEN
}

\section{RESUMEN}

En este trabajo se presentan brevemente tres experiencias de profesoras universitarias que en el marco del aislamiento social preventivo y obligatorio, al igual que el resto de la docencia argentina, debimos "mudar" nuestras prácticas y nuestros saberes de la modalidad presencial a una plataforma digital.

En la primera parte, expondremos acerca del concepto saberes del oficio del enseñar y de experiencia, luego relataremos nuestras prácticas como docentes en el escenario que impone la cuarentena, en el que describiremos las situaciones vividas y la incertidumbre que por momentos nos acompaña, para señalar qué aspectos de ese saber del oficio, saber-hacer, fueron puestos en tensión y cómo los estamos resolviendo.

Finalmente, nos preguntamos si las transformaciones subjetivas que se producen en este tiempo lograrán generar impacto en las organizaciones universitarias y en nuestras prácticas o, si por el contrario, una vez ubicados en una "nueva normalidad", trataremos de volver a lo que nos es conocido.

\section{ABSTRACT}

In this work, three experiences of university professors are briefly presented that, in the context of preventive and compulsory social isolation, like the rest of Argentine teaching, we had to "move" our practices and our knowledge of the face-to-face modality to a digital platform.

In the first part, we will expose about the concept of knowledge of the profession of teaching and experience, then we will relate our practices as teachers in the scenario imposed by quarantine, in which we will describe the situations experienced and the uncertainty that accompanies us at times, to point out what aspects of that knowledge of the trade, know-how, were put into tension and how we are solving them.

Finally, we ask ourselves whether the subjective transformations that take place during this time will have an impact on university organizations and on our practices or, on the contrary, once located in a "new normal", we will try to return to what is us. known.

PALABRAS CLAVE

Saberes del oficio,

Enseñanza,

Experiencia,

Transformaciones

KEY WORDS

Knowledge of the trade,

Teaching,

Experience,

Transformations 


\section{PRESENTACIÓN}

A partir de la experiencia vivida en estos tiempos de pandemia, tres profesoras que desarrollamos el oficio de enseñar en la universidad pública relatamos y reflexionamos en el marco del aislamiento social preventivo y obligatorio.

Hemos elegido reflexionar tomando dos ejes conceptuales como saberes del oficio y experiencia, para poder pensar-narrar las experiencias vividas respondiendo a tres preguntas que a nuestro criterio, abarcan la complejidad de los procesos en los que estamos involucradas.

Esta reflexión sería incompleta si no mantenemos la mirada atenta y crítica para comprender también cómo se juegan en esta pandemia los intereses por sostener el statu quo, a la vez que se tensionan con otras decisiones que impone la inmediatez.

La estructura del trabajo es la siguiente: en la primera parte, expondremos acerca del concepto saberes del oficio del enseñar y de experiencia, luego relataremos nuestras prácticas como docentes en el escenario que impone la cuarentena, en el que describiremos las situaciones vividas y la incertidumbre que por momentos nos acompaña, para señalar qué aspectos de ese saber del oficio, saber-hacer, fueron puestos en tensión y cómo los estamos resolviendo.

Finalmente, nos preguntamos si las transformaciones subjetivas que se producen en este tiempo lograrán generar impacto en las organizaciones universitarias y en nuestras prácticas o, si por el contrario, una vez ubicados en una "nueva normalidad", trataremos de volver a lo que nos es conocido. 


\section{CONCEPTOS Y EXPERIENCIAS EN PANDEMIA}

El concepto saberes de oficio es utilizado para referirse a aquellos saberes que se producen a lo largo de las trayectorias profesionales, pero que no siempre son tenidos en cuenta en el marco de diseños o programas institucionales de formación (Alliaud y Suárez, 2011). Son saberes que responden al "saber hacer" $y$ "saber ser", más allá del conocimiento teórico y formal y se acercan más a la experiencia de la práctica, tienen características propias y se relacionan con otros campos del saber. Indagar sobre los saberes de oficio posibilita desentrañar la cocina donde se elabora la práctica pedagógica: la previa, el acontecimiento pedagógico y las evaluaciones posteriores con sus aciertos y desaciertos. De este modo, se comienzan a poner en evidencia las capacidades y habilidades que los docentes van desarrollando a lo largo de su carrera para poder adaptarse a los acelerados y profundos cambios culturales, tecnológicos, políticos y económicos. También permite reconstruir la identificación de problemáticas socioeducativas que van emergiendo en ese proceso y la toma de decisiones situadas en contextos educativos singulares.

Indagar sobre los saberes de oficio posibilita desentrañar la cocina donde se elabora la práctica pedagógica: la previa, el acontecimiento pedagógico y las evaluaciones posteriores con sus aciertos y desaciertos.

De este modo, al decir de Suárez y Metzdorff (2018), documentar los saberes de oficio posibilita

(...) conocer buena parte de la experiencia de formación y de las trayectorias profesionales de las y los docentes implicados, de los saberes pedagógicos y supuestos sobre la enseñanza que informan sus prácticas, de sus recorridos vitales y experiencias laborales, de sus certezas, dudas y preguntas, de sus inquietudes, deseos y logros (...) (p.58).

Los saberes de oficio se constituyen en el curriculum alternativo, polifónico, plural, múltiple sobre el estar siendo docente en un contexto determinado.

Atendiendo al contexto de pandemia que estamos atravesando, utilizaremos el concepto saberes del oficio docente, para dar cuenta de las contradicciones de nuestro saberhacer con la nueva realidad educativa que se nos impone.

Atendiendo al contexto de pandemia que estamos atravesando, utilizaremos el concepto saberes del oficio docente, para dar cuenta de las contradicciones de nuestro saberhacer con la nueva realidad educativa que se nos impone. 


\subsection{EL CONTEXTO DE PANDEMIA}

El contexto sociopolítico, cultural y sanitario actual está caracterizado, podríamos decir regido, por la incertidumbre. El aislamiento social preventivo y obligatorio instala la necesidad "abrupta" -definición política- de utilizar las tecnologías para sostener la escolaridad de las y los niños/as y jóvenes en todo el país, del mismo modo que desafía a legitimar nuestro trabajo.

Esta situación desnuda y revela las prácticas pedagógicas cotidianas, en el sentido que plantea A. Heller (1970) la vida cotidiana, cargada de saberes de distinta procedencia. Algunas de esas prácticas, están rutinizadas y mecanizadas; otras, en cambio, pretenden ser reflexivas y críticas. Pero, el actual momento de pausa, de excepción (Agamben, 2004) de lo conocido, la "mudanza" de la educación al hogar y la necesaria utilización de tecnologías desafía u "obliga" -según la vivencia de cada docente- a repensar la propia práctica pedagógica para poder adecuarla a formatos virtuales, que hasta este momento, no habíamos practicado en su totalidad, sin ninguna alternativa de presencialidad. Probablemente la vivencia de cada docente pueda explicarse por sus singulares trayectorias, las tradiciones formativas, el contexto institucional, las experiencias de formación continua y tantas otras variables que ponen en evidencia la riqueza de la reconstrucción del saberhacer en el contexto actual.

Entendemos que el actual momento vivido es singular -sin dudasporque deja al desnudo lo habitual, lo cotidiano, lo rutinario, lo mecanizado, lo que sabemos hacer y que de golpe hay que pensarlo- revisarlo-reordenarlo para ponerlo en acto sobre una plataforma virtual.

Esta realidad es la que nos moviliza hoy a la narración de nuestra experiencia, con la convicción de que la autoreflexión pueda ser testimonio de algunas- entre otras- vivencias, decisiones y acciones pedagógicas en un tiempo de pandemia. Hemos elaborado algunos interrogantes guías que ordenaran el relato:

- ¿Cómo atravesamos emocionalmente el lugar del no saber? ¿Cómo resolvimos la situación de virtualidad total? ¿A quiénes recurrimos? ¿De qué modo la institución acompaña este tiempo de nuevos aprendizajes y formatos pedagógicos?

- ¿Cuáles son las dificultades socioculturales y económicas con las qué se encuentran las y los estudiantes al llevar adelante la práctica? ¿De qué modo lo resuelven? ¿Qué evaluación hacemos de esa experiencia?

- ¿Cómo vivimos la experiencia de comunión (al estar en casa) sobre crianza/conviviencia full time familiar y trabajo docente? ¿Qué reflexión se puede hacer al respecto? 


\section{NUESTRAS EXPERIENCIAS}

Nos proponemos compartir situaciones vividas que llamamos experiencias, desde un concepto particular de la misma, no desde el sentido de la experticia o el saber construido a través del tiempo. Tomamos y seguimos la propuesta de Jorge Larrosa que define la experiencia como "eso que me pasa, que supone un acontecimiento, algo que no depende de mí, ni de mi saber, ni de mi poder, ni de mi voluntad" (Larrosa, 2009, p.14). El acontecimiento supone un"principio de subjetividad", en el que el sujeto es capaz y se permite que algo le pase en sus palabras, en sus ideas, en sus sentimientos, en sus representaciones. Es siempre experiencia de alguien, que es propia y que cada uno "padece" de un modo único, singular, particular y propio, lo que implica reflexividad y apropiación sensible única del sujeto. Desde este concepto y desde su riqueza es que presentamos las experiencias que nos pasan en este tiempo.

Nos proponemos compartir situaciones vividas que llamamos experiencias, desde un concepto particular de la misma, no desde el sentido de la experticia o el saber construido a través del tiempo. Tomamos y seguimos la propuesta de Jorge Larrosa que define la experiencia como "eso que me pasa, que supone un acontecimiento, algo que no depende de mí, ni de mi saber, ni de mi poder, ni de mi voluntad

Las experiencias que se presentan, corresponden a tres profesoras que nos desempeñamos en la Universidad Pública. Trabajamos en el Departamento de Educación y las tres narraciones se realizan sobre/ desde cátedras de formación docente.

Resulta importante mencionar, dado el contexto de producción de las experiencias, que desde hace muchos años ésta Universidad desarrolló el área de educación a distancia (así denominado en los primeros tiempos) y de educación virtual (a partir del desarrollo de las TIC y su contribución al campo educativo) y que se ha esforzado por ofrecer circuitos de formación y capacitación a los docentes de todas las áreas disciplinares en esta modalidad educativa. Los cursos formativos siempre han sido gratuitos, en el marco del enriquecimiento de la carrera académica de los docentes. Su realización o no, ha quedado a criterio de las posibilidades y disponibilidades de cada uno de los agentes del sistema.

Hoy, ante la "mudanza" del formato presencial al virtual, la UNICEN cuenta con una plataforma bajo el sistema Moodle, dependiente del rectorado y descentralizado en cada una de las facultades y escuelas secundarias dependientes de ella. 


\section{Experiencia 1: La incertidumbre como acontecimiento de lo cotidiano}

Mi desempeño docente durante el primer cuatrimestre del calendario académico es en la cátedra de la asignatura Política Educativa, espacio curricular que cursan estudiantes de los dos Profesorados que se dictan en la Facultad, de Comunicación Social y de Antropología. Comparto el trabajo con dos colegas con las que habitualmente, cada año, ordenamos y programamos la cursada en término de encuentros /clases denominados tradicionalmente como "teóricos" y "prácticos". En esta oportunidad nos sorprendió la medida de aislamiento cuando aún no habíamos comenzado con el desarrollo de la cursada.

Este fue el primer hecho que modificó e impactó en el trabajo docente, reprogramando una tarea, la de pensar y diagramar un cronograma de clases y temas, de forma virtual y con la incertidumbre instalada en nuestras vidas. Una incertidumbre compartida no solo con las colegas de trabajo sino también con los y las estudiantes, con el desconcierto que esta situación provocaba -y provoca- en la cotidianeidad de todos nosotros.

Conocerles a través de la pantalla e intercambiar las presentaciones en un ámbito casi desconocido para mí, fue impactante (si bien he asistido a charlas, cursos y seminarios virtuales, no ha sido desde mi lugar de enseñante). Propusimos un encuentro semanal, en el lugar de los dos que habitualmente hacíamos en la presencialidad, a través del software de videolladas Zoom. Además, "subimos" al aula virtual la propuesta temática con una orientación de lectura y habilitar un grupo de Whatsapp para estar más cerca ante cualquier requerimiento. Poner a disposición cualquier herramienta que hiciera falta para achicar la distancia de no encontrarnos en el aula, en el pasillo de la facultad.

Propusimos un encuentro semanal, en el lugar de los dos que habitualmente hacíamos en la presencialidad, a través del software de videolladas Zoom. Además, "subimos" al aula virtual la propuesta temática con una orientación de lectura y habilitar un grupo de Whatsapp para estar más cerca ante cualquier requerimiento. Poner a disposición cualquier herramienta que hiciera falta para achicar la distancia de no encontrarnos en el aula, en el pasillo de la facultad.

La preparación de las clases implica una experiencia totalmente nueva, escribir, contar explicar, nombrar las ideas de cada tema de manera tal que pueda ser comprendido por los alumnos. Siempre con la inquietud de ser lo suficientemente clara, no enredarme, considerar todos los temas importantes y sobre todo no abrumar con la información. Sabiendo que nos falta la palabra en vivo, el encuentro físico, el lugar compartido de aula para dar lugar a la pregunta, las miradas, las interrupciones, los gestos que complementan el acto pedagógico. En 
este sentido, ha sido fundamental el compartir las nuevas decisiones del hacer con las compañeras de cátedra, pensar y ejecutar otras formas para facilitar la tarea a los estudiantes.

Con el transcurso de la semana los estudiantes fueron refiriendo sus sensaciones ante esta contingencia, el cansancio que provoca seguir con los requerimientos de cada signatura a través de la virtualidad, el reordenamiento que también ellas y ellos tuvieron que realizar en sus vidas, poder administrar sus tiempos, los espacios físicos y herramientas tecnológicas con el resto de los integrantes de sus familias. Esa situación fue compartida con cada una de nosotras que también tuvimos que adaptarnos en este compartir lugares, computadoras, horarios con nuestra familia.

Esta situación tan inédita y abrupta nos enfrenta a preguntas y dudas que replantean la tarea de enseñar. La reflexión sobre la práctica se vuelve una constante ya que ninguna de las tareas que realizamos es absolutamente conocida de antemano y menos aún "sus resultados" pueden ser predecibles, todo se vuele más incierto, cada acción se ve mediatizada por variables que en la presencialidad, que eran conocidas para nosotras. Estamos transitando un tiempo que sin dudas "se hará experiencia" en cada uno de nosotros, nos transformará y transformará nuestras vidas, pero sobre todo deberá transformar los sentidos de la experiencia de la enseñanza y todo lo que eso implica. Re-pensar la formación, los sentidos del saber a enseñar y del saber hacer para poder ser, transmitir y re-significar los saberes, es el desafío que tenemos por delante.

Esta situación tan inédita y abrupta nos enfrenta a preguntas y dudas que replantean la tarea de enseñar. La reflexión sobre la práctica se vuelve una constante ya que ninguna de las tareas que realizamos es absolutamente conocida de antemano y menos aún "sus resultados" pueden ser predecibles, todo se vuele más incierto, cada acción se ve mediatizada por variables que en la presencialidad, que eran conocidas para nosotras.

\section{Experiencia 2: una experiencia de humanización en una virtualidad con pandemia}

Soy profesora -en el primer cuatrimestre de cada año académico- de la materia Didáctica de la Comunicación Social, en el profesorado de Comunicación Social. Hace dos años realicé el curso formativo para uso de la plataforma virtual Moodle. Nunca imaginé que estaba aprendiendo a usar las herramientas que hoy me posibilitan seguir desempeñando mi trabajo de enseñar y que con ello favorecería el derecho de las y los estudiantes, de aprender.

Junto al JTP, distribuimos los contenidos en las 16 clases del cuatrimestre. Al inicio, las mismas daban cuenta de un desarrollo conceptual en armonía metodológica con las herramientas de la plataforma. 
La inquietud por ver a los estudiantes hizo que de inmediato invite a encontrarnos a través de Zoom, para vernos, conocernos, contar cómo estábamos viviendo el primer período de aislamiento social preventivo y obligatorio que ya avizorábamos que no duraría tan solo quince días. Pero...encontré placas con nombre y no rostros, en la mayoría de los casos. ¿Por qué me hacen esto? -pensé. Y conversando con colegas, supe que no me hacían nada, que la mayoría prefería esa forma de estar de incógnito, sin miradas ni gestualidades, sin que penetráramos la intimidad de su casa.

encontré placas con nombre y no rostros, en la mayoría de los casos. ¿Por qué me hacen esto? -pensé. Y conversando con colegas, supe que no me hacían nada, que la mayoría prefería esa forma de estar de incógnito, sin miradas ni gestualidades, sin que penetráramos la intimidad de su casa.

A medida que pasó el tiempo supe también que la mayoría no tenía Internet en su casa; en muchos hogares había una sola computadora compartida y la mayor parte de las actividades se hacía desde los teléfonos celulares. Ese dato me impactó. Yo no podría escribir ni una oración con coherencia usando solo el celular -pensé-. Y también se pusieron en jaque mis supuestos sobre el trabajo sincrónico y el asincrónico.

¿Tengo que compartir el espacio y el tiempo para que haya enseñanza y aprendizaje? -pensaba- No tengo espacio para desarrollar todas las ideas que han danzado por mi mente desde esos momentos iniciales, que recorrieron mi biografía como aprendiz y como enseñante, para encontrarle la vuelta a una práctica de la enseñanza que tantas veces he recitado y de la que me encuentro teóricamente convencida.

Me sacudió la otredad, su presencia en la virtualidad. Esto es: el sujeto que aprende como elemento de la tríada de la Didáctica, como objeto de estudio de la Psicología del Aprendizaje, como actor de la Política Educativa, se manifestaba en toda su diversidad, su complejidad y su realidad. Compartir la pantalla o sentir la ausencia de quienes ya sabía que podrían estar pero no estaban, ya no me dejarían armar las clases con el distanciamiento del inicio.

Mientras yo cocinaba, limpiaba la casa (tareas que habitualmente no realizo) o hablaba con mis hijas que están estudiando en otra ciudadpensaba en las clases para que fueran un espacio valioso de pensamiento, que conlleven fuerza para generar autonomía a las/los futuros docentes, que dejen huellas en sus memorias. Me pensaba a mí misma sintiendo este momento tan vital cuando todo estaba suspendido, todo estaba en estado de excepción (Agamben, 2004). Porque no solo es virtualidad: es virtualidad con pandemia, combinación que vuelve la relación más compleja aún. La incertidumbre del futuro, la "nueva normalidad" de la que nos hablan atraviesa también con su incertidumbre este presente.

En este momento que -como dijimos antes- deja al desnudo lo habi- 
tual, lo mecanizado, lo específico del oficio docente, se puso en primer plano la humanidad. Yo creo que estoy transitando una experiencia de humanización, largamente proclamada y pocas veces hecha práctica en la docencia.

\section{Experiencia 3: Cuando el relato de la experiencia sensible supera el aná- lisis científico...}

Soy ayudante diplomada de Didáctica especial de la Antropología y el Taller de Prácticas de la Enseñanza de la Comunicación y la Antropología. El momento transitado impacta significativamente en la tarea pedagógica que llevo adelante desde hace más de 10 años.

La primera decisión que tomamos con mis compañeras de cátedra fue dialogar con los y las estudiantes mediante comunicaciones vía email, con el propósito de diagnosticar la disponibilidad del servicio de internet. Luego elaboramos un primer comunicado en el que informamos la modalidad de trabajo. Allí se definió sostener la modalidad de Tutorial virtual utilizando el email, el grupo de WhatsApp, Hangouts y/o Zoom, la frecuencia de encuentros semanales y se explicitó el envío de los materiales de lectura y las indicaciones de trabajos prácticos (TP) con fechas de entrega y modalidad (grupal y/o individual).

En esa misma comunicación, luego de explicitar los modos de trabajo los invitamos a reflexionar y analizar el video de Pablo Yafe "Sobre ser estudiantes en tiempos de COVID-19" con dos objetivos: por un lado habilitarlos a expresar sus primeras impresiones sobre el material y además relatar sus sentires y preocupaciones en este contexto que trastoca nuestra cotidianeidad. El segundo objetivo fue el análisis del material audiovisual (estructura, narrativa, recursos, objetivos...).

Cuando recibimos las producciones nos sorprendimos porque pudimos leer los "sentires" de cada uno de ellos/ellas. Notamos que la producción los corrió del lugar de estudiantes de las disciplinas de formación, para expresar las sensaciones vividas en este tiempo. Pudimos leer sus posicionamientos políticos partidarios, los modos en que irrumpió la pandemia en su cotidianeidad, los miedos, las incertidumbres.

Cuando recibimos las producciones nos sorprendimos porque pudimos leer los "sentires" de cada uno de ellos/ellas. Notamos que la producción los corrió del lugar de estudiantes de las disciplinas de formación, para expresar las sensaciones vividas en este tiempo. Pudimos leer sus posicionamientos políticos partidarios, los modos en que irrumpió la pandemia en su cotidianeidad, los miedos, las incertidumbres.

El segundo objetivo de la actividad quedó al margen, el análisis se redujo, a tan solo un párrafo o dos como máximo, de una descripción técnica escasamente complejizadora.

El encuentro virtual amplió el deseo de expresión de la experiencia 
transitada. Allí, al abrir las "puertas" de su hogar se mostraron más distantes del análisis disciplinar, se "desnudaron" en la intimidad de sus emociones y admitieron "no ver" con profundidad la consigna 2.

Esta experiencia, en tanto docentes formadoras, en un contexto que también nos moviliza, nos lleva a preguntarnos cuál es el sentido de nuestra práctica pedagógica en un contexto como el actual. Observamos que cada planificación no logra ajustarse a los tiempos programados, las lecturas no logran hacerse con la profundidad que pretendemos y las explicaciones de los y las estudiantes redundan en la complejidad del tránsito de su cotidianeidad en este tiempo. Se expresan y muestran cansados de leer mediados por una pantalla, muchas veces no "los vemos" porque pausan el video "para garantizar mejor conectividad", otras nos encontramos con sus niños y niñas.

Observamos que cada planificación no logra ajustarse a los tiempos programados, las lecturas no logran hacerse con la profundidad que pretendemos y las explicaciones de los y las estudiantes redundan en la complejidad del tránsito de su cotidianeidad en este tiempo. Se expresan y muestran cansados de leer mediados por una pantalla, muchas veces no "los vemos" porque pausan el video "para garantizar mejor conectividad", otras nos encontramos con sus niños y niñas.

Del mismo modo que en ellos y ellas irrumpe la pandemia, en nosotras también lo hace. Muchas veces nos vemos corrigiendo una producción por el celular mientras bañamos a nuestros niños/niñas o nos sentimos corriendo en nuestro hogar para organizar el/los encuentros virtuales.

Claramente esta experiencia redefine la organización del tiempo, el espacio, lo público, lo privado, el tiempo de producción y de ocio, porque la matriz cultural occidental, capitalista y neoliberal se cuela en la organización rutinaria de nuestra vida. El tiempo productivo, la disciplina, la eficacia, la disponibilidad laboral parece -muchas vecesprimar sobre la experiencia de estar viviendo un tiempo único y finito, que sin dudas dejará huellas imborrables en nuestra experiencia de vida y práctica laboral.

\section{Reflexiones}

En estas reflexiones pretendemos anudar el sentido que producen las tres experiencias narradas, con las hipotéticas transformaciones que puedan producirse, una vez salidos de este estado de excepción en que nos colocó la pandemia y habitemos la incierta "nueva normalidad".

Hoy las tres experiencias nos marcan la centralidad de la emoción, la experiencia sensible y el redescubrimiento de la humanidad que habita en nosotros y los otros.

El momento singular y excepcional que nos toca vivir, como suje- 
tos, en el devenir del tiempo se torna un acontecimiento en la medida en que somos capaces de permitirnos, porque podemos, reflexionar y hacer de este tiempo una apropiación sensible única. ¿Podemos apropiarnos del acontecimiento porque tenemos tiempo para reflexionar, al estar suspendida la vertiginosidad de nuestra vida cotidiana "normal"? La virtualidad nos acercó a una dimensión enunciada pero poco vivida sobre los estudiantes, sobre su centralidad en el acto pedagógico atendiendo a la complejidad del contexto sociocultural y económico por el que transitan. Esa misma virtualidad no es una virtualidad cualquiera, es una virtualidad con/en pandemia, cuestión que también pone en suspenso el tema de la enseñanza y evaluación de los contenidos para permitirnos pensar, quizá, en un acto pedagógico que hoy tiene más de social y humanitario que de conocimiento.

La experiencia pedagógica mediada tecnológicamente como única forma de existencia puso en tensión, también la borrosa relación entre lo público y lo privado, tanto para las y los estudiantes como para nosotras, las docentes. De algún modo, el imperativo de "hacer seguir girando la rueda" del sistema educativo recayó sobre la docencia y, nuevamente, pusimos la fuerza del trabajo.

\section{La experiencia pedagógica mediada tecnológicamente como única forma de existencia puso en tensión, también la borrosa relación entre lo público y lo privado, tanto para las y los estu- diantes como para nosotras, las docentes.}

Nuestra intención es poder aproximarnos a los saberes del oficio reflexivamente, para comprender los efectos subjetivos que ésta situación inédita tiene en nuestras prácticas y toda su potencialidad resignificada.

También queremos referir a algunos aspectos estructurales que este tiempo devela y nos rebela:

- Los modos y estrategias originarias del sistema capitalista, norteurocéntico y neocolonial respecto de la necesidad de control biopolítico ante lo imprevisto mediante normas, explicaciones científicas, que se proponen construir seguridades mediante anticipaciones técnicas, lineales, ordenadas, utilitaristas sobre la proyección de futuro.

- En pos del "orden", la "rutinización", las políticas públicas y educativas en particular, no han dado tiempo para pausar y "oir" lo que sucede de la "piel para adentro" (Kush: 1962) lo emocional, lo imprevisto, el desorden, el caos, las pasiones. Situación que sí ha emergido en los encuentros de aprendizaje real, situado. Allí los y las estudiantes, los y las docentes mostramos interés por expresar las emociones vividas en este tiempo. Aspecto que consideramos resulta sumamente significativo poder recuperar para que el "volver a la normalidad" no se reduzca 
a una evaluación -disciplinar- de lo que aprendieron o no en este contexto singular, sino una posibilidad de resignificación de las prácticas pedagógicas en los diversos contextos educativos nacionales.

Es por estas cuestiones sintetizadas en las reflexiones, que ponemos en tensión también las posibilidades y límites de una transformación subjetiva en constante tensión relacional con una superestructura que se hace presente con toda su fuerza, para mantener el statu quo. 


\section{BIBLIOGRAFIA}

Agamben, G. (2005). “Estado de excepción". Homo sacer, II, I. Buenos Aires: Adriana Hidalgo editora.

Alliaud, A. y Suárez, D. (2011). "El saber de la experiencia. Experiencias pedagógicas, narración y subjetividad en la trayectoria profesional de los docentes". Disponible en: http://www.filo.uba.ar/contenidos/investigacion/institutos/lice/ANUARIO_2008/textos/32_Andrea_Alliaud.pdf

Heller, A. (1970). “Sociología de la vida cotidiana”. Barcelona, España: Península

Kush, R. (1962). "Introducción a América", en América Profunda, Obras completas, Tomo 2, Rosario: Ed. Ross

Larrosa, J. (2009). "Experiencia y Alteridad en Educación", en Experiencia y Alteridad en Educación Larrosa, J y Skliar, C (compiladores) Rosario: Homo Sapiens, pp. 13-44

Suárez, D. y Metzdorff, V. (2018). "Narrar la experiencia educativa como formación. La documentación narrativa y el desarrollo profesional de los docentes". En: Revista de Educación. Serie Indagaciones n² 28.

Salit, C. (2011). "Enseñar didáctica. Sentidos construidos desde una propuesta de formación situada". Ponencia concurso.

Scarafía, S. y Constenla, M. (2011). “Docencia universitaria virtual y demandas de escritura: Un nuevo desafío de alfabetización". Revista VEsC -Año 2- Número 3- pp. 29-42.

Terigi, F. (2008). "El saber pedagógico frente a la crisis de la monocronía". En Frigerio, G. y Diker, G. (Comp.). Educar: saberes alterados. Buenos Aires: CLACSO Coediciones - Del estante editorial.

Yazyi, M. (2015). “Reflexión final foro ¿Desde qué lugar/es (en el mundo) tomó la palabra? Discutir sentidos del equipo coordinadores y tutores. Materia Prácticas de Enseñanza con TIC, Especialización Superior en Educación Primaria y TIC, Instituto Nacional de Formación Docente, Ministerio de Educación de la Nación. Argentina. 